\title{
Slug mediates nasopharyngeal carcinoma radioresistance via downregulation of PUMA in a p53-dependent and -independent manner
}

\author{
TING XU, BIN FAN, CHUNJIANG LV and DAJIANG XIAO \\ Department of Otolaryngology, The Second People's Hospital of Wuxi, Wuxi, Jiangsu 214002, P.R. China
}

Received December 14, 2014; Accepted March 2, 2015

DOI: $10.3892 /$ or.2015.3877

\begin{abstract}
Slug is involved in the radioresistance and chemoresistance of several types of cancers. In the present study, we first studied the effect of Slug on the radioresistance of nasopharyngeal carcinoma (NPC). We established radioresistant CNE-2 cells (CNE-2-RES) by exposing CNE-2 cells to gradually increasing doses of irradiation (IR). We used lentiviral infection technique to stably knock down Slug and then studied the effects in vitro and in vivo. Western blotting and RT-PCR were applied to detect the protein and mRNA expression in NPC cells or xenograft tumor tissues, respectively. Colony forming assay was applied to detect the cell survival after IR. As a result, CNE-2-RES cells were successfully established, CNE-2-RES cells showed relatively higher expression of Slug, higher expression of p53 and lower expression of PUMA. Following inhibition of Slug, the radiosensitivity of NPC was enhanced both in vitro and in vivo. Slug inversely regulated PUMA and p53 expression in both CNE-2 and CNE-2-RES cells. Animal experiments showed the same trend of protein expression as the in vitro results. In conclusion, our study demonstrated that Slug overexpression in CNE-2-RES cells may result in the radioresistance of cells. Slug mediates CNE-2 radioresistance via downregulation of PUMA in both a p53-dependent and p53-independent manner.
\end{abstract}

\section{Introduction}

Nasopharyngeal carcinoma (NPC) has an unusual geographical and ethnic distribution, and is one of the most common cancers in Southeast Asia and Southern China, especially

Correspondence to: Dr Chunjiang Lv or Professor Dajiang Xiao, Department of Otolaryngology, The Second People's Hospital of Wuxi, 68 Zhong Shan Road, Wuxi, Jiangsu 214002, P.R. China

E-mail: 15861671322@126.com

E-mail: guiming.zhu@gmail.com

Key words: nasopharyngeal carcinoma, radioresistance, Slug, p53 upregulated modulator of apoptosis, p53 among individuals of Cantonese origin (1). The highest incidence of this disease is found in this area (peaking at $50 / 100,000$ people/year) (2). Although detection of NPC by imaging and advanced radiotherapy techniques have led to an improvement in the management and treatment of NPC, radioresistance remains a serious barrier to successful treatment in many cases $(3,4)$. Radiotherapy in cancer therapy directly or indirectly damages DNA and induces apoptosis. Defects in the apoptotic machinery can lead to radioresistance (5). The exact molecular mechanism involved in NPC radioresistance remains poorly understood. Therefore, it is essential to investigate the potential mechanism of NPC radioresistance.

The Slug protein belongs to the Snail superfamily of zinc finger transcription factors (6). It is closely related to transcriptional repressors implicated in embryonic development, where they have been shown to be vital for the formation of the mesoderm and neural crest through epithelial-mesenchymal transition (7). Studies have shown that in malignant tumors, Slug not only participates in the regulation of carcinogenesis, invasiveness and metastasis in various cancers (8-13), but also has an anti-apoptotic effect (14-18). However, few studies have been undertaken to assess whether Slug can be involved in the radioresistance of cancers. Recently, Findlay et al reported that calcitriol (1 $\alpha, 25$-dihydroxyvitamin D3) enhanced radiation sensitivity in colorectal cancer, but overexpression of Slug inhibited this effect (19). It was reported that in cholangiocarcinoma and melanoma cells, Slug inhibition can enhance radiosensitivity by upregulation of the activity of p53 upregulated modulator of apoptosis (PUMA), which has been shown to be involved in the control of apoptosis $(20,21)$. In ovarian cancer cells, Slug was found to promote radioresistance by antagonizing p53-mediated apoptosis (22). However, the function of Slug associated with radioresistance in NPC has never been previously investigated.

In the present study, we successfully established radioresistant CNE-2 cells (CNE-2-RES) by exposing CNE-2 cells to gradually increasing doses of irradiation (IR). It was demonstrated that upregulation of Slug expression contributed to the radioresistance of CNE-2-RES cells which was associated with downregulated PUMA expression. By inhibition of Slug, the radiosensitivity of NPC was enhanced both in vitro and in vivo. These results have implications for the treatment of NPC. 


\section{Materials and methods}

Cell culture and the establishment of radioresistant CNE-2-RES cells. The poorly differentiated NPC cell line CNE-2 and the high differentiated NPC cell line CNE-1 (Shanghai Bogoo Biological Technology Co., Ltd., Shanghai, China) were cultured in Roswell Park Memorial Institute (RPMI)-1640 medium (Invitrogen Life Technologies, Carlsbad, CA, USA) supplemented with $10 \%$ fetal bovine serum (FBS), with $100 \mathrm{IU} / \mathrm{ml}$ penicillin and streptomycin at $37^{\circ} \mathrm{C}$ in a humidified atmosphere with $5 \% \mathrm{CO}_{2}$. The selection procedure of CNE-2-RES cells was performed as previously described (23). The parental CNE-2 cells, which were used as the control, were treated according to the same procedure except for the IR step. CNE-2-RES cells were cultured in the same culture medium as the CNE-2 cells. Exponentially growing cells were used for all experiments.

Cell viability and colony forming assay. A Cell Counting Kit-8 (Beyotime Biotechnology Co., Ltd., Shanghai, China) was used according to the manufacturer's instructions to determine the growth of both cell lines. Cells were plated onto 96-well plates at a density of $2 \times 10^{3}$ cells/well in triplicate. After $12 \mathrm{~h}$ of culture, the cells were exposed to different doses of IR (2, 4, 6, 8 and $10 \mathrm{~Gy})$. Absorbance values were expressed as percentages relative to the controls. For the colony forming assay (24), cells were seeded into 6-well culture plates at $1 \times 10^{3}$ cells/well for $12 \mathrm{~h}$ and were exposed to IR with doses ranging from 2 to $10 \mathrm{~Gy}$. The cells were then cultured for an additional 14 days. Next, the cells were washed twice with phosphate-buffered saline, fixed with methanol/ acetic acid (3:1; v/v) (both from SunShine Biotechnology Co., Ltd., Nanjing, China) and stained with $0.5 \%$ crystal violet (C3886; Sigma Chemical Co., St. Louis, MO, USA). The number of colonies was counted under a microscope. The number of surviving colonies (a colony was defined as $>50$ cells) was counted under a microscope (Nikon TE2000; Nikon Corporation, Japan).

Silencing of Slug by shRNA. Three pairs of shRNAs targeting different regions of the human Slug transcript (GenBank: U97060) and 1 control shRNA were designed and synthesized (Invitrogen Life Technologies). They were cloned into the pLentiLox 3.7 lentiviral vector between $H p a \mathrm{I}$ and XhoI. The packaged lentiviruses which showed the highest knockdown efficiency of Slug mRNA in the two cell lines was used for the experiments. Cells were seeded into 6-well plates at a density of $1.5 \times 10^{3}$ cells ( $70 \%$ confluency) and were infected with control lentiviral shRNA and lentiviral shRNA Slug which were referred to as negative and LV-sh-Slug. The cells were assayed by RT-PCR and western blotting on the second day after infection.

Silencing of p53 by siRNA. In order to silence the p53 gene, p53 siRNA (sc-45917; Santa Cruz Biotechnology, Inc., Santa Cruz, CA, USA) was used to transfect the CNE-2-RES cells. After infection with LV-sh-Slug, CNE-2-RES cells were then transfected with p53 siRNA according to the protocol. The transfection efficiency was detected by western blotting as described below.
Reverse transcription-PCR. Total RNA was isolated using Trizol according to a standard protocol from cells of each group. Total RNA was treated with DNase I (Invitrogen Life Technologies) to remove the contaminating genomic DNA. PCR analysis was conducted using the One-Step reverse transcription-PCR kit (Invitrogen Life Technologies). Actin was used as an internal control. The following primers were used: Slug sense, 5'-CATGCCTGTCATACCACAAC-3' and antisense, 5'-GGTGTCAGATGGAGGAGGG-3'; PUMA sense, 5'-GACGACCTCAACGCACAGTA-3' and antisense, 5'-AGG AGTCCCATGATGAGATTGT-3'; p53 sense, 5'-AATCTC ACCCCATCCCACAC-3' and antisense, 5'-GACCCTGAG CATAAAACAAGTCT-3'; actin sense, 5'-TGATGGGTGTGA ACCACGAG-3' and antisense, 3'-TTGAGTCGCAGGA GACAACC-5'. The PCR conditions were as follows: $95^{\circ} \mathrm{C}$ for $3 \mathrm{~min}$, followed by 40 cycles of $95^{\circ} \mathrm{C}$ for $30 \mathrm{sec}, 55^{\circ} \mathrm{C}$ for $20 \mathrm{sec}$ and $72^{\circ} \mathrm{C}$ for $20 \mathrm{sec}$. The melting curve program was $95^{\circ} \mathrm{C}$ for $15 \mathrm{sec}, 60^{\circ} \mathrm{C}$ for $15 \mathrm{sec}$, and $20 \mathrm{~min}$ for warming. The final extension was at $95^{\circ} \mathrm{C}$ for $15 \mathrm{sec}$. PCR was performed under a quantitative PCR instrument (Bio-Rad, Shanghai, China).

Western blotting. Western blot analyses were performed as described previously (25). Briefly, total of $50 \mu \mathrm{g}$ proteins were extracted from the cells of each group. After electrophoresis, transmembrane and blocking, the blotted membranes were incubated with primary monoclonal anti-Slug (sc-166476, 1:500); polyclonal anti-PUMA (sc-20534, 1:500) and monoclonal anti-p53 (sc-126, 1:1,000) antibodies, which were purchased from Santa Cruz Biotechnology, Inc., at $4^{\circ} \mathrm{C}$ overnight. Subsequent to being washed, the membranes were incubated with HRP-labeled anti-goat or anti-mouse (Boster Biological Engineering Co., Ltd., Wuhan, China) for $1 \mathrm{~h}$ at room temperature. Bands were visualized by employing the BeyoECL Plus Detection system (Beyotime Institute of Biotechnology, Jiangsu, China). The intensity of protein fragments was quantified with Quantity One software (4.5.0 Basic; Bio-Rad, Hercules, CA, USA) and represented as the densitometric ratio of the targeted protein to $\beta$-actin. Cell protein lysates were assayed in triplicate.

Xenograft tumor experiments. Male, 4- to 6-week-old BALB/c nude mice were purchased from Shanghai Animal Center (Shanghai, China). The mice were then observed daily for their diet consumption, stools and mental state. The body weight was measured every three days. On the day of tumor cell inoculation, tumor cells at $70-80 \%$ confluency were trypsinized and resuspended in FBS-free culture medium. Xenograft tumors were established by subcutaneous injection of $2 \times 10^{6} \mathrm{NPC}$ cells (CNE-2, CNE-2-RES, CNE-2-RES LV-sh-Slug) into the groin area of the 4- to 6-week-old male nude mice ( $n=6$, a total of 2 subgroup). Two weeks later, the mice in one subgroup were exposed to an IR dose of $4 \mathrm{~Gy}$, while the other did not. The lengths and widths of the tumors were measured with Vernier calipers and calculated using the following formula: Tumor volume $=$ length $\mathrm{x}$ width ${ }^{2} \times 0.5$. The mice were sacrificed 2 weeks later in accordance with institutional regulations for animal experiments. The use of animals in the present study complied with the Guide for the Care and Use of Laboratory Animals. The study was approved by the Institutional Animal Care and Use Committee, Wuxi, Jiangsu, China. The tissue 

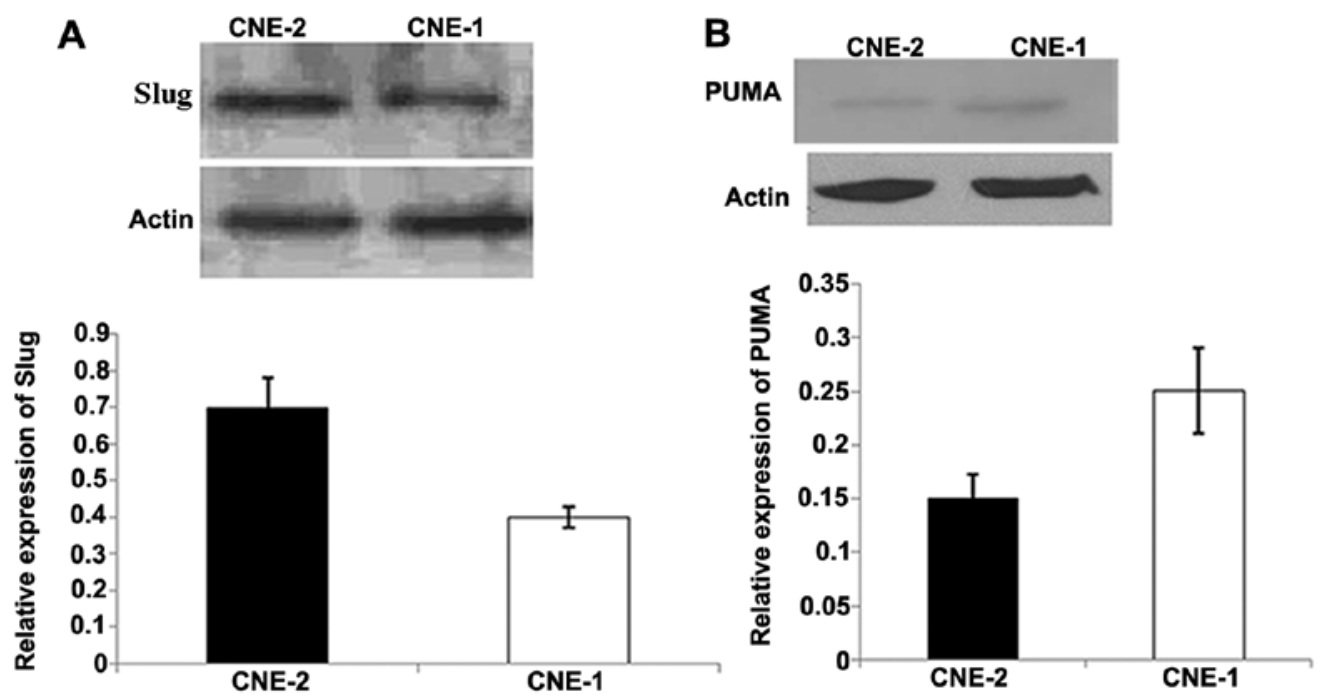

Figure 1. Slug and PUMA proteins were detected by western blotting in the CNE-1 and CNE-2 cells (normalized to $\beta$-actin). (A) Results of the western blotting and graph of Slug expression in the CNE-1 and CNE-2 cells. (B) Results of western blotting and the graph of PUMA expression in the CNE-1 and CNE-2 cells. Values are the mean $\pm \mathrm{SD}$ of three independent experiments.
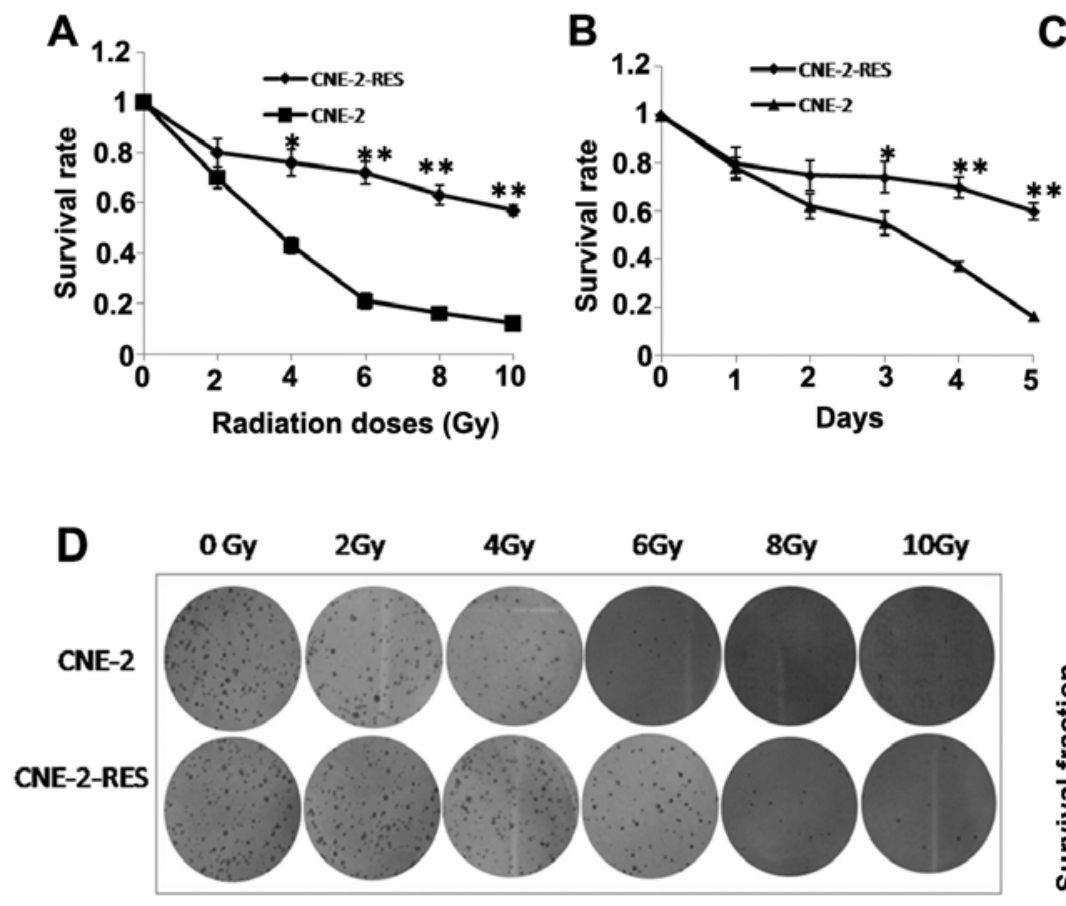

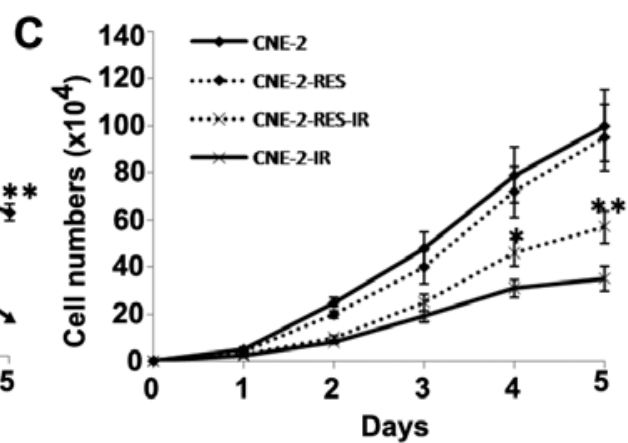

Radiation doses (Gy)

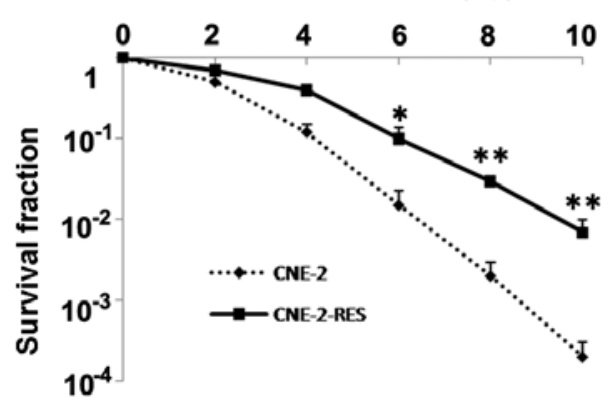

Figure 2. Radioresistant CNE-2 cells (CNE-2-RES) are established and validated. The survival rates for CNE-2-RES and CNE-2 cells at (A) different irradiation (IR) doses $(0,2,4,6,8$ and $10 \mathrm{~Gy})$ and at (B) different time-points $(1,2,3,4$ and 5 days) were determined using a CCK-8 assay. (C) The cell growth curves of CNE-2-RES and CNE-2 cells exposed or not exposed to 4-Gy IR at different time-points (1,2,3, 4 and 5 days). (D) A representative image of colony formation in CNE-2-RES and CNE-2 cells exposed or not exposed to different doses of IR (0, 2, 4, 6, 8 and 10 Gy) after 14 days (left). Survival fractions of CNE-2-RES and CNE-2 cells were obtained from the results of the colony forming assays (right). The results are the average of three independent experiments \pm standard deviation (SD) $\left({ }^{*} \mathrm{P}<0.05,{ }^{* *} \mathrm{P}<0.01\right)$.

sections were viewed at x100 magnification, and images were captured with a digital camera.

Statistical analysis. The SPSS 11.5 for Windows statistical analysis software package (SPSS, Inc., Chicago, IL, USA) was employed for the analysis of data. The Student's t-test and Mann-Whitney U test were used for the statistical analysis of data. A $\mathrm{P}<0.05$ was considered to indicate a statistically significant difference.

\section{Results}

Slug expression in the NPC cells. We detected the mRNA expression of Slug in the CNE-1 and CNE-2 cells. As shown in 
A

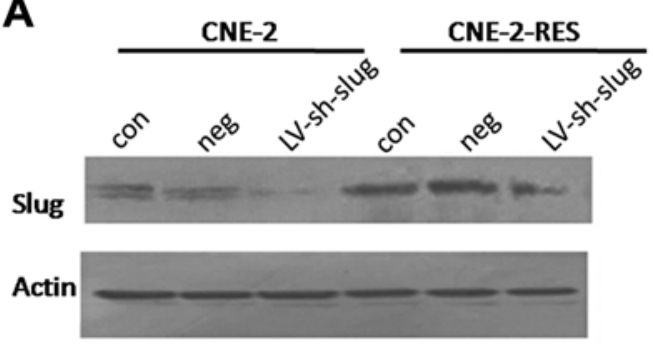

B

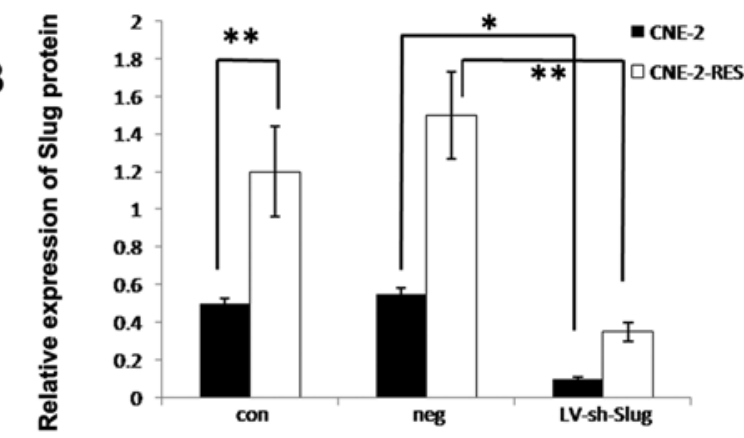

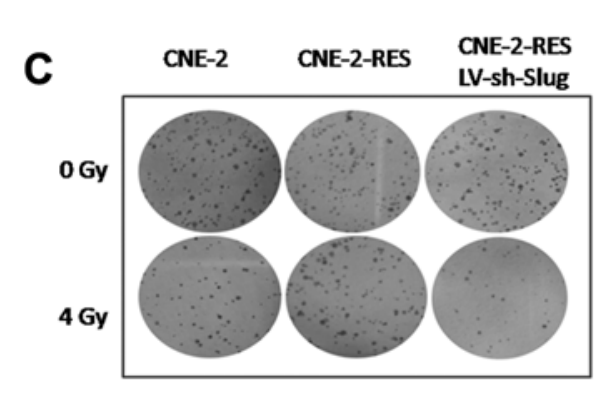

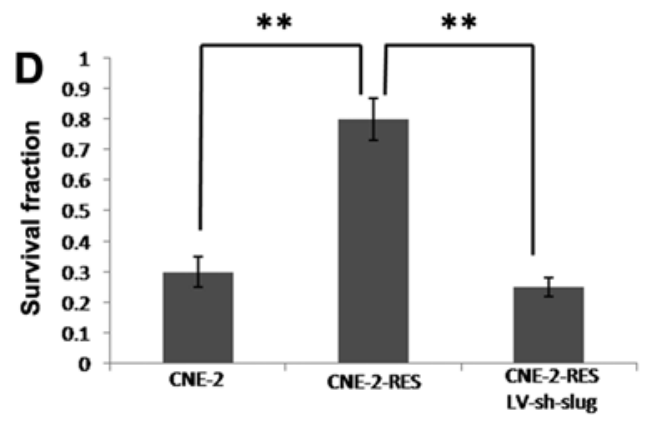

Figure 3. CNE-2-RES and CNE-2 cells are infected with LV-sh-Slug, and the protein level of Slug is detected. After infection, Slug expression was significantly inhibited. The radioresistant CNE-2-RES cells showed significantly increased Slug expression when compared with the CNE-2 cells. Knockdown of Slug in the CNE-2-RES cells resulted in increased sensitivity to irradiation. (A and B) Western blot analysis results of Slug protein expression. The results are the average of three independent experiments \pm standard deviation (SD) $\left({ }^{*} \mathrm{P}<0.05,{ }^{* * *} \mathrm{P}<0.01\right.$ comparing the two groups). (C) A representative image of colony formation in the different cell groups (CNE-2, CNE-2-RES and CNE-2-RES cells infected with LV-sh-Slug) with or without a 4-Gy dose of irradiation. (D) Quantitative measurement of the survival fraction in each cell group. The results are the average of three independent experiments \pm standard deviation (SD) (** $\mathrm{P}<0.01$ vs. CNE-2-RES).

A

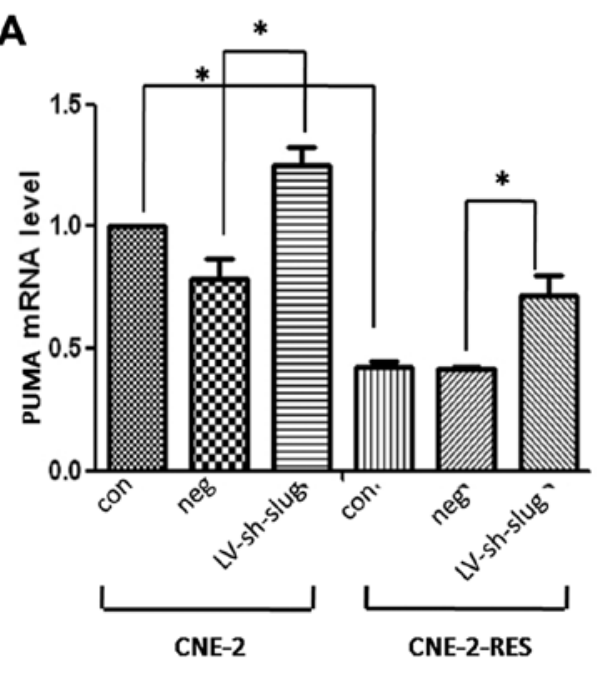

B
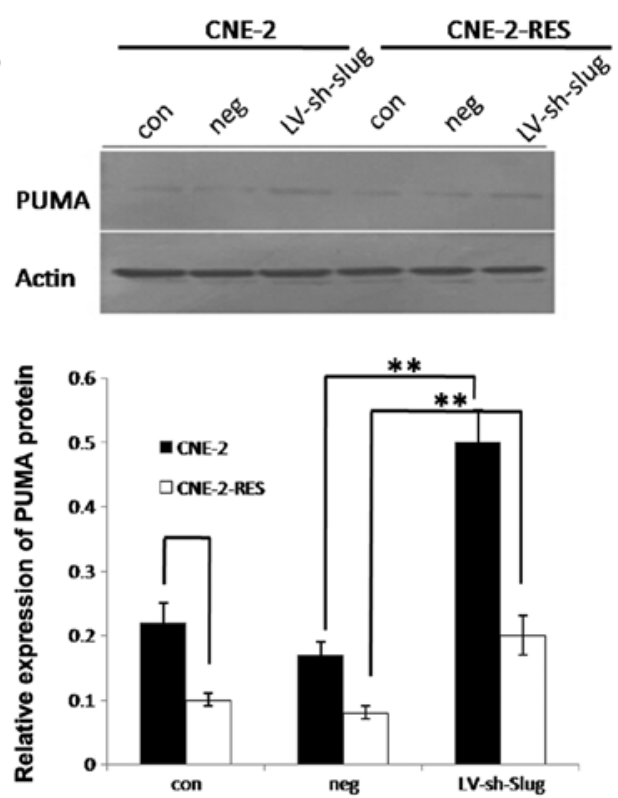

Figure 4. The mRNA and protein of PUMA are detected in CNE-2-RES and CNE-2 cells after knockdown of Slug. After infection, PUMA expression was significantly increased in both cell lines. The radioresistant CNE-2-RES cells showed significantly decreased PUMA expression when compared with the CNE-2 cells. (A) Real time RT-PCR results of PUMA mRNA expression. (B) Western blotting results of PUMA protein expression. The results are the average of three independent experiments \pm standard deviation (SD) ( $\mathrm{P}<0.05,{ }^{* * *} \mathrm{P}<0.01$ comparing the two groups).

Fig. 1, CNE-2 cells showed relatively higher expression of Slug mRNA than that in the CNE-1 cells. Thus, we chose CNE-2 cells for the following experiments.
Radioresistant CNE-2-RES cell line is successfully established and its radioresistant capacity is validated. The cell radioresistant capacity was validated by CCK-8 and colony 


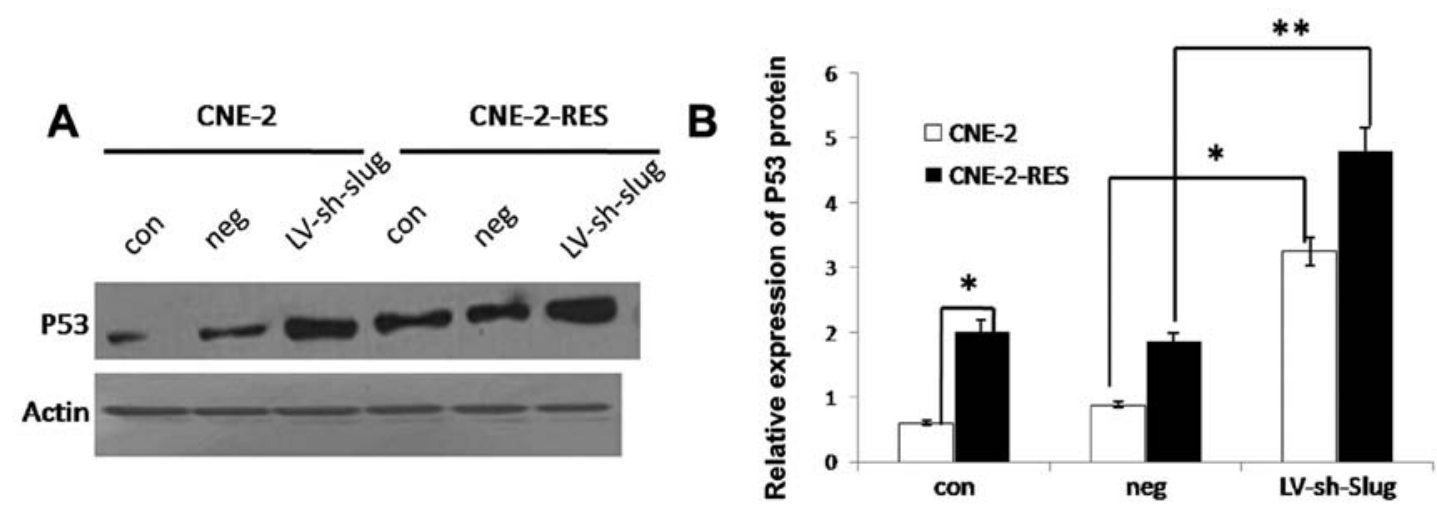

C

D
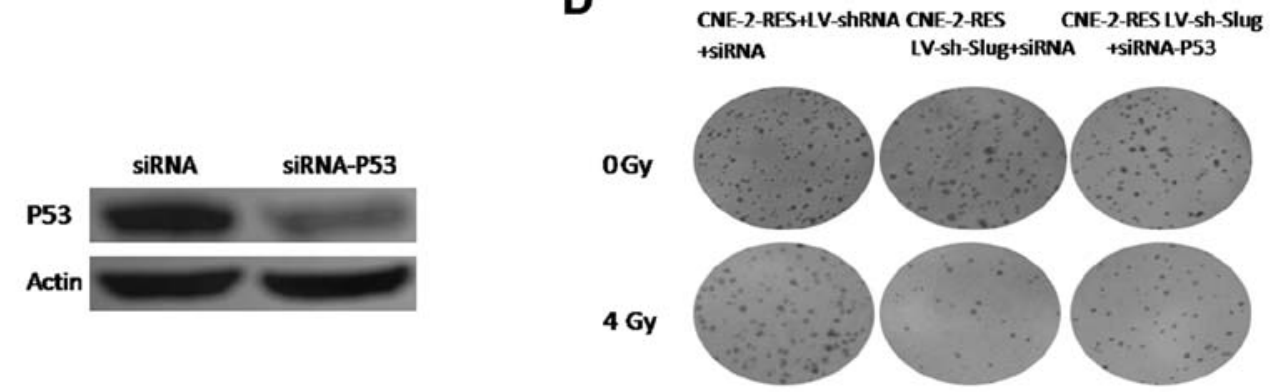

Figure 5. Slug regulates p53 expression in the CNE-2 and CNE-2-RES cells. (A and B) p53 was detected in the CNE-2-RES and CNE-2 cells after knockdown of Slug. After infection, p53 expression was significantly increased in both cell lines. The radioresistant CNE-2-RES cells showed significantly increased p53 expression when compared to that in the CNE-2 cells. (C) p53 siRNA was transfected to silence p53 expression in the LV-sh-Slug-infected CNE-2-RES cells. After transfection, p53 protein in the CNE-2-RES cells was significantly inhibited. (D) Colony forming assay showed that following a 4-Gy dose of IR, inhibition of p53 in the LV-sh-Slug infected CNE-2-RES cells partly restored the ability of radioresistance under $4 \mathrm{~Gy}$ of IR $\left({ }^{*} \mathrm{P}<0.05\right.$, ${ }^{* *} \mathrm{P}<0.01$ comparing the two groups).

forming assays. The survival rates of CNE-2-RES cells following gradually increasing doses of IR $(0,2,4,6,8$ and $10 \mathrm{~Gy}$ ) were higher than those of the CNE- 2 cells. The two cell lines were then exposed to a 4-Gy dose of IR, and at various time-points $(1,2,3,4$ and 5 days) we observed that the survival rates of the CNE-2-RES cells were significant higher than those of the CNE-2 cells (Fig. 2A and B). The effect of IR on NPC cell growth was examined under a 4-Gy dose of IR by CCK-8, After a 4-Gy dose of IR, at various time-points (1, 2, 3, 4 and 5 days), the growth rate of the CNE-2-RES cells was significantly inhibited compared with the parental cells (Fig. 2C). A colony forming assay indicated that a greater number of CNE-2-RES cell colonies survived when compared with the CNE-2 cells (Fig. 2D).

Slug expression is increased in the radioresistant CNE-2-RES cells. We detected the expression of Slug protein by western blotting. When the CNE-2 cells acquired the ability of resistance, Slug protein expression was significantly increased $(\mathrm{P}<0.05$, Fig. $3 \mathrm{~A}$ and $\mathrm{B})$.

PUMA expression is decreased in the radioresistant CNE-2-RES cells. Results showed that when the CNE-2 cells acquired the ability of resistance, both the PUMA mRNA and protein levels were significantly decreased $(\mathrm{P}<0.05$, Fig. $4 \mathrm{~A}$ and $\mathrm{B})$.

Knockdown of Slug in the CNE-2-RES cells increases their sensitivity to IR. Slug protein was significantly inhib- ited in the CNE-2-RES cells following knockdown of Slug (Fig. 3A and B); the infection efficiency was $89.38 \pm 2.1 \%$. As shown by the colony forming assay, following a 4-Gy dose of IR, inhibition of Slug in the CNE-2-RES cells led to a decreased number of surviving clones compared to the negative control $(25.9 \pm 5.2$ vs. $79.4 \pm 10.1 \%$; $P<0.01$, Fig. $3 C$ and $D)$.

Slug regulates PUMA expression in the CNE-2 and CNE-2-RES cells. After Slug was knocked down in both cell lines (CNE-2 and CNE-2-RES), we detected the expression of PUMA by real time RT-PCR and western blotting. The results showed that both the expression levels of PUMA mRNA and protein were significantly increased in both cell lines $(\mathrm{P}<0.05$, Fig. 4A and B). This result revealed that Slug might promote the radioresistant ability of CNE-2-RES cells by downregulating PUMA.

Slug mediates CNE-2-RES cell radioresistance via the p53-independent pathway. We further detected expression of the tumor-suppressor gene p53. Surprisingly, the radioresistant CNE-2-RES cells showed significantly increased p53 expression when compared with that in the CNE-2 cells. This result demonstrated that the p53-independent pathway may play an important role in Slug-induced radioresistance in CNE-2-RES cells.

Slug regulates p53 expression in the CNE-2 and CNE-2-RES cells. We detected p53 after downregulation of Slug. Knockdown 

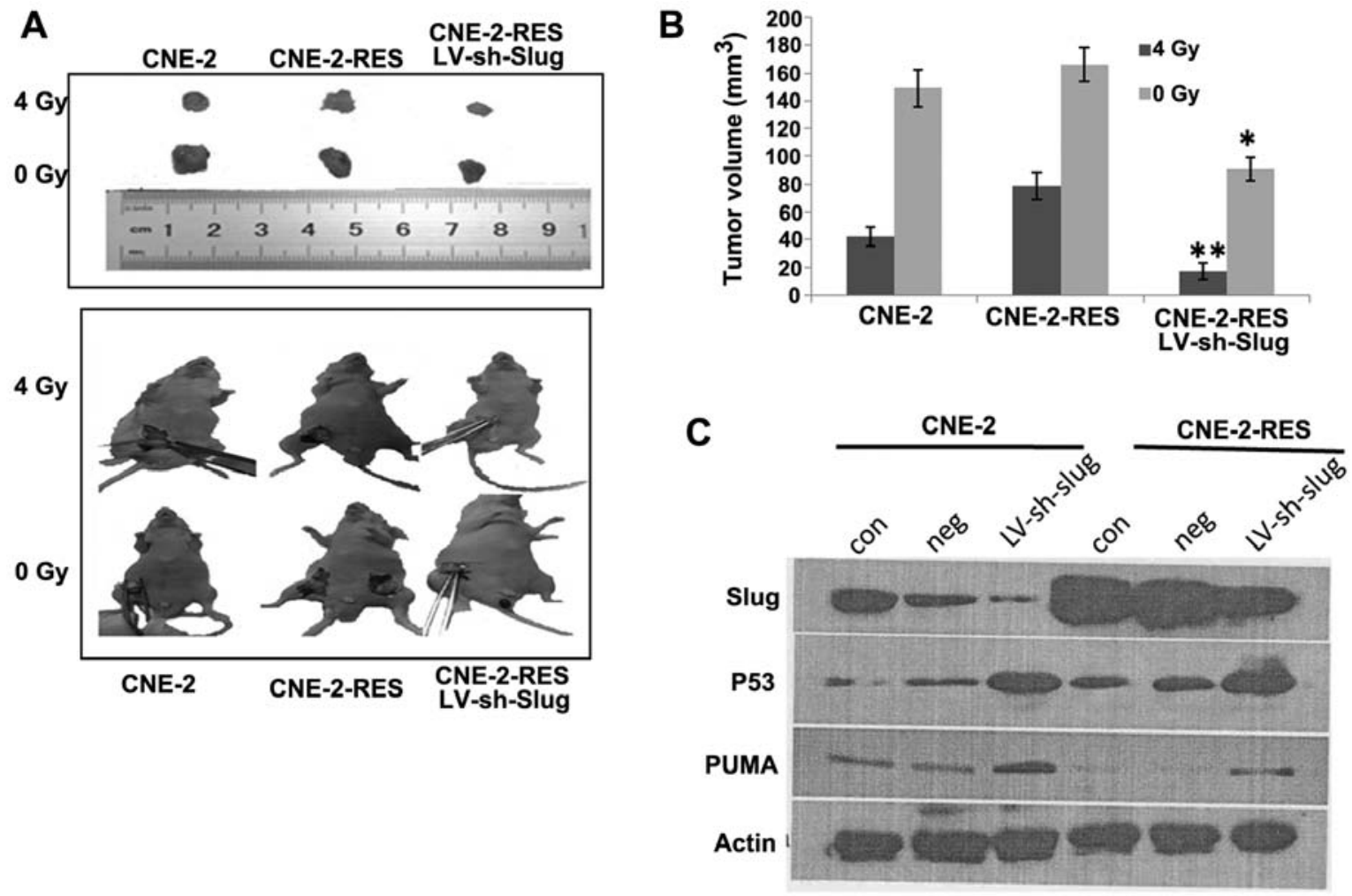

Figure 6. Slug shRNA plus radiation mediate xenograft tumor growth and protein expression in tumor tissues. (A) CNE-2 and CNE-2-RES cells were subcutaneously injected into the groin area of 4- to 6-week-old male nude mice. Two weeks later, the mice were exposed to 0 and a 4-Gy dose of IR. An additional 2 weeks later, images of the xenograft tumors and tissue sections were captured at x100 magnification with a digital camera. (B) Graph of the tumor volume of the various xenografts ( ${ }^{*} \mathrm{P}<0.05,{ }^{* *} \mathrm{P}<0.01 \mathrm{vs}$. CNE-2-RES cells). (C) Western blotting for Slug, PUMA and p53 in the xenograft tumor tissues from mice treated with CNE-2, CNE-2-RES, CNE-2 LV-shRNA-Slug and CNE-2-RES LV-shRNA-Slug cells using specific antibodies for these molecules.

of Slug resulted in increased p53 expression (Fig. 5A and B). To further demonstrate that Slug mediates CNE-2 radioresistance via downregulation of PUMA in a p53-dependent manner, we used p53 siRNA to silence p53 expression in the LV-sh-Slug infected CNE-2-RES cells. The results showed that after transfection, p53 protein in the CNE-2-RES cells was significantly inhibited; the transfected efficiency was $85.26 \pm 3.9 \%$ (Fig. 5C), It was shown by colony forming assay that following a 4-Gy dose of IR, inhibition of p53 in the LV-sh-Slug-infected CNE-2-RES cells partly restored the ability of radioresistance under $4 \mathrm{~Gy}$ of IR. The number of surviving clones in the CNE-2-RES+LV-shRNA+siRNA, CNE-2-RES+LV-sh-Slug+siRNA-p53 and CNE-2-RES $+\mathrm{LV}$-sh-Slug+siRNA cells were 76.32 $\pm 9.5,29.1 \pm 4.8$ and $53.4 \pm 6.1 \%$, respectively, $(\mathrm{P}<0.01$, Fig. 5D). These results suggest that Slug mediates $\mathrm{CNE}-2$ radioresistance partly via downregulation of PUMA in a p53-dependent manner.

Knockdown of Slug decreases the radioresistance of NPC xenografts in vivo. After 4 weeks, when receiving no radiation, the xenograft tumors that were established by subcutaneous injection of CNE-2-RES, LV-sh-Slug cells showed tumor regression of $45.3 \pm 3.6 \%$ compared with the CNE-2-RES group; there was no difference between the CNE-2 and CNE-2-RES groups ( $\mathrm{P}>0.05)$. After receiving a 4-Gy dose of radiation at 2 weeks, the tumor volumes of xenografts that were established by subcutaneous injection of CNE-2, CNE-2-RES and CNE-2-RES LV-shRNA-Slug cells decreased by $42.3 \pm 7,78.6 \pm 9.3$ and $17.4 \pm 5.6 \%$, respectively. These findings indicated that when CNE-2 cells acquired radioresistant ability, the corresponding xenograft tumors also acquired radioresistance. Yet, following knockdown of Slug, the radioresistance of the CNE-2-RES xenografts was significantly reduced (Fig. 6A and B).

We further detected Slug, PUMA and p53 protein in the xenograft tumor tissues. CNE-2-RES xenograft tumor tissues showed relatively higher Slug and p53 expression and lower PUMA expression than the CNE-2 xenograft tumor tissues. Following knockdown of the Slug gene, the corresponding xenograft tumor tissues showed higher p53 and PUMA expression (Fig. 6C). These results were consistent with the previous in vitro experiment.

\section{Discussion}

Resistance of NPC to radiotherapy is a major problem in cancer treatment (26). Slug, a snail family transcription factor, is a suppressor of PUMA, which has been shown to be involved in the radioresistance and chemoresistance of several types of cancers (19-22). In the present study, we successfully established radioresistant CNE-2-RES cells to study the role of Slug in NPC. When CNE-2 cells acquired the ability of radioresistance, Slug expression was significantly upregulated. Colony forming assay further showed that knockdown of Slug significantly increased the radiosensitivity of CNE-2-RES cells following a 4-Gy dose of IR. These results suggest that 
Slug overexpression in CNE-2-RES cells may result in the radioresistance of cells.

It is known that Slug can repress PUMA gene transcription in many types of cells (19-22,27). PUMA-induced apoptosis mainly occurs through activation of the tumor-suppressor protein p53 (28). PUMA-induced apoptosis may also be promoted independently of $\mathrm{p} 53$ activation by other stimuli, such as oncogenic stress, growth factors and/or cytokine withdrawal and kinase inhibition, ER stress, altered redox status, ischemia, immune modulation, and infection (28). But the function of PUMA in p53-independent apoptosis remains to be fully elucidated (28). You et al (29) and Adlakha and Saini (30) domonstrated that nuclear-activated FOXO3A binds the PUMA promoter regardless of the p53 genotype, thereby demonstrating that FOXO3A can act directly on the PUMA promoter in a $\mathrm{p} 53$-independent manner.

In the present study, radioresistant CNE-2-RES cells showed downregulated PUMA expression and upregulated p53 expression. The change in PUMA which was inconsistent with p53 suggests that Slug might mediate radiation resistance in CNE-2-RES cells via inhibition of PUMA but not antagonizing p53-dependent apoptosis. There is probably some p53-independent signaling pathways involved in the Slug/PUMA axis associated with apoptosis in CNE-2 cells, or Slug might act through another signaling pathway other than the PUMA/P53 axis. Our results were not consistent with an ovarian cancer report, which showed that Slug mediated radiation resistance mainly by p53-dependent apoptosis (22). Following knockdown of Slug, we detected PUMA and p53 expressions in both the CNE-2 and CNE-2-RES cell lines. Slug inversely regulated PUMA and p53 expression in both cell lines. Knockdown of p53 in the LV-sh-Slug-infected CNE-2-RES cells partly restored the ability of radioresistance under 4 Gy of IR. These results suggest that Slug mediates CNE-2 radioresistance partly via downregulation of PUMA in a p53-dependent manner. The result demonstrates that the Slug/PUMA/p53 axis does exit and act in the Slug induced radiation resistance of CNE-2-RES cells, but Slug-induced radiation resistance is the result of joint action of many signaling pathways. Slug-induced radioresistance is mediated both by antagonizing p53-mediated apoptosis and not in CNE-2-RES cells. Yet, identification of the exact signaling pathway involved in the p53-independent apoptosis needs further investigation.

Notably, Slug expression was relatively higher in the CNE-2 cells than that in the CNE-1 cells, and PUMA expression is relatively lower in $\mathrm{CNE}-2$ cells than that in $\mathrm{CNE}-1$ cells. To the best of our knowledge, CNE-2 is relatively more radiosensitive than CNE-1 (31); this phenomenon was inconsistent with our above results. We believe that this may be because CNE1 and CNE2 are two different types of NPC cells. The degree of differentiation and other features are not the same, thus the two cell lines are not comparable. Moreover, the radiosensitivity of NPC cells is determined by the effects of multiple factors, and is not limited to Slug.

Slug-induced radioresistance was further verified in animal experiments. When CNE-2 cells acquired radioresistant ability, the corresponding xenograft tumors also acquired radioresistance. But following knockdown of Slug, the radioresistance of the CNE-2-RES xenografts was significantly reduced. The trends of protein expressed in the animal tissues were similar to the results we detected in vitro.

Taken together, our results demonstrated that Slug is a valuable radioresistance-associated biomarker and a promising therapeutic target in the management of NPC. Slug inhibition may be useful for chemoprevention and/or therapy of NPC. Yet, the p53-independent signaling pathway needs further study in subsequent research.

\section{Acknowledgements}

This study was supported by grants from the General Program of Health Bureau of Wuxi City (no. ML201314) and the Key Program of Nanjing Medical University (no. 2014NJMUZD034).

\section{References}

1. Lo KW, Chung GT and To KF: Deciphering the molecular genetic basis of NPC through molecular, cytogenetic, and epigenetic approaches. Semin Cancer Biol 22: 79-86, 2012.

2. Wee JT, Ha TC, Loong SL and Qian CN: Is nasopharyngeal cancer really a 'Cantonese cancer'? Chin J Cancer 29: 517-526, 2010.

3. Jemal A, Siegel R, Xu J and Ward E: Cancer statistics, 2010. CA Cancer J Clin 60: 277-300, 2010.

4. Yeh SA, Tang Y, Lui CC, Huang YJ and Huang EY: Treatment outcomes and late complications of 849 patients with nasopharyngeal carcinoma treated with radiotherapy alone. Int J Radiat Oncol Biol Phys 62: 672-679, 2005.

5. Coultas L and Strasser A: The molecular control of DNA damage-induced cell death. Apoptosis 5: 491-507, 2000.

6. Nieto MA: The Snail superfamily of zinc-finger transcription factors. Nat Rev Mol Cell Biol 3: 155-166, 2002.

7. Ros MA, Sefton M and Nieto MA: Slug, a zinc-finger gene previously implicated in the early patterning of the mesoderm and the neural crest, is also involved in chick limb development. Development 124: 1821-1829, 1997.

8. Lee HJ, Jeng YM, Chen YL, Chung L and Yuan RH: Gas6/Axl pathway promotes tumor invasion through the transcriptional activation of Slug in hepatocellular carcinoma. Carcinogenesis 35: 769-775, 2014.

9. Shih JY and Yang PC: The EMT regulator slug and lung carcinogenesis. Carcinogenesis 32: 1299-1304, 2011.

10. Shirley SH, Greene VR, Duncan LM, Torres Cabala CA, Grimm EA and Kusewitt DF: Slug expression during melanoma progression. Am J Pathol 180: 2479-2489, 2012.

11. Wu WS, Heinrichs S, Xu D, Garrison SP, Zambetti GP, Adams JM and Look AT: Slug antagonizes p53-mediated apoptosis of hematopoietic progenitors by repressing puma. Cell 123: 641-653, 2005.

12. Huang $\mathrm{CH}$, Yang WH, Chang SY, Tai SK, Tzeng CH, Kao JY, $\mathrm{Wu} \mathrm{KJ}$ and Yang MH: Regulation of membrane-type 4 matrix metalloproteinase by SLUG contributes to hypoxia-mediated metastasis. Neoplasia 11: 1371-1382, 2009.

13. Zhang KJ, Wang DS, Zhang SY, Jiao XL, Li CW, Wang XS, Yu QC and Cui HN: The E-cadherin repressor slug and progression of human hilar cholangiocarcinoma. J Exp Clin Cancer Res 1: 88, 2010.

14. Inukai T, Inoue A, Kurosawa H, Goi K, Shinjyo T, Ozawa K, Mao M, Inaba T and Look AT: SLUG, a ces-1-related zinc finger transcription factor gene with antiapoptotic activity, is a downstream target of the E2A-HLF oncoprotein. Mol Cell 4: 343-352, 1999.

15. Cha HS, Bae EK, Ahn JK, Lee J, Ahn KS and Koh EM: Slug suppression induces apoptosis via Puma transactivation in rheumatoid arthritis fibroblast-like synoviocytes treated with hydrogen peroxide. Exp Mol Med 42: 428-436, 2010.

16. Dhasarathy A, Phadke D, Mav D, Shah RR and Wade PA: The transcription factors Snail and Slug activate the transforming growth factor-beta signaling pathway in breast cancer. PLoS One 6: e26514 2011.

17. Kurrey NK, Amit K and Bapat SA: Snail and Slug are major determinants of ovarian cancer invasiveness at the transcription level. Gynecol Oncol 97: 155-165, 2005. 
18. Martin TA, Goyal A, Watkins G and Jiang WG: Expression of the transcription factors snail, slug, and twist and their clinical significance in human breast cancer. Ann Surg Oncol 12: 488-496, 2005

19. Findlay VJ, Moretz RE, Wang C, Vaena SG, Bandurraga SG, Ashenafi M, Marshall DT, Watson DK and Camp ER: Slug expression inhibits calcitriol-mediated sensitivity to radiation in colorectal cancer. Mol Carcinog (Suppl 53): E130-E139, 2014.

20. Zhang K, Zhang B, Lu Y, Sun C, Zhao W, Jiao X, Hu J, Mu P, $\mathrm{Lu} \mathrm{H}$ and Zhou C: Slug inhibition upregulates radiation-induced PUMA activity leading to apoptosis in cholangiocarcinomas. Med Oncol (Suppl 28): S301-S309, 2011.

21. Arienti C, Tesei A, Carloni S, Ulivi P, Romeo A, Ghigi G, Menghi E, Sarnelli A, Parisi E, Silvestrini R, et al: SLUG silencing increases radiosensitivity of melanoma cells in vitro. Cell Oncol (Dordr) 36: 131-139, 2013.

22. Kurrey NK, Jalgaonkar SP, Joglekar AV, Ghanate AD, Chaskar PD, Doiphode RY and Bapat SA: Snail and slug mediate radioresistance and chemoresistance by antagonizing p53-mediated apoptosis and acquiring a stem-like phenotype in ovarian cancer cells. Stem Cells 27: 2059-2068, 2009.

23. Li G, Liu Y, Su Z, Ren S, Zhu G, Tian Y and Qiu Y: MicroRNA-324-3p regulates nasopharyngeal carcinoma radioresistance by directly targeting WNT2B. Eur J Cancer 49: 2596-2607, 2013.

24. Franken NA, Rodermond HM, Stap J, Haveman J and van Bree C: Clonogenic assay of cells in vitro. Nat Protoc 1: 2315-2319, 2006.
25. Xu T, Yu CY, Sun JJ, Liu Y, Wang XW, Pi LM, Tian YQ and Zhang X: Bone morphogenetic protein-4-induced epithelialmesenchymal transition and invasiveness through Smad1-mediated signal pathway in squamous cell carcinoma of the head and neck. Arch Med Res 42: 128-137, 2011.

26. Lee N, Xia P, Quivey JM, Sultanem K, Poon I, Akazawa C, Akazawa P, Weinberg V and Fu KK: Intensity-modulated radiotherapy in the treatment of nasopharyngeal carcinoma: an update of the UCSF experience. Int J Radiat Oncol Biol Phys 53: 12-22, 2002.

27. Perez-Mancera PA, Gonzalez-Herrero I, Perez-Caro $M$, Gutiérrez-Cianca N, Flores T, Gutiérrez-Adán A, Pintado B, Sánchez-Martín M and Sánchez-García I: SLUG in cancer development. Oncogene 24: 3073-3082, 2005.

28. Yu J and Zhang L: PUMA, a potent killer with or without $\mathrm{p} 53$. Oncogene (Suppl 27): S71-S83, 2008.

29. You H, Pellegrini M, Tsuchihara K, Yamamoto K, Hacker G, Erlacher M, Villunger A and Mak TW: FOXO3a-dependent regulation of Puma in response to cytokine/growth factor withdrawal. J Exp Med 203: 1657-1663, 2006.

30. Adlakha YK and Saini N: miR-128 exerts pro-apoptotic effect in a 553 transcription-dependent and -independent manner via PUMA-Bak axis. Cell Death Dis 4: e542, 2013.

31. Qu Y, Zhang H, Zhao S, Hong J and Tang C: The effect on radioresistance of manganese superoxide dismutase in nasopharyngeal carcinoma. Oncol Rep 23: 1005-1011, 2010. 